\title{
Entre tradition et modernité
}

L'avenir de l'enseignement supérieur au Mexique

The future of higher education in Mexico

El futuro de la enseñanza superior en México

\section{Salvador Malo}

Traducteur : Nuria Aligant

\section{OpenEdition}

\section{Journals}

Édition électronique

URL : http://journals.openedition.org/ries/141

DOI : 10.4000/ries. 141

ISSN : 2261-4265

Éditeur

Centre international d'études pédagogiques

Édition imprimée

Date de publication : 1 septembre 2007

Pagination : $39-49$

ISBN : 978-2-85420-570-

ISSN : $1254-4590$

Référence électronique

Salvador Malo, «Entre tradition et modernité », Revue internationale d'éducation de Sèvres [En ligne],

45 | septembre 2007, mis en ligne le 22 juin 2011, consulté le 03 mai 2019. URL : http://

journals.openedition.org/ries/141; DOI : 10.4000/ries.141 


\title{
Entre tradition et modernité
}

\section{L'avenir de l'enseignement supérieur au Mexique *}

\author{
Salvador Malo
}

\section{LES QUESTIONNEMENTS}

Dans la seconde moitié du $\mathrm{XX}^{\mathrm{e}}$ siècle, le tissu social, démographique, politique et économique du Mexique a connu de multiples et profondes mutations qui ont rythmé et accentué les transformations vécues également dans le domaine éducatif. Le développement démographique, industriel et commercial, associé à d'autres facteurs ainsi qu'aux politiques mises en œuvre, a accru la demande d'accès à l'enseignement supérieur.

À partir de 1950, et en écho à la demande exprimée par la société, l'ensemble des institutions, des enseignants, des étudiants, ainsi que les autres acteurs qui façonnent le système d'enseignement supérieur mexicain (SESM), ont enclenché une dynamique extraordinaire et toujours d'actualité. Aujourd'hui, le SESM a été multiplié par 80 , avec un doublement de ses effectifs tous les neuf ans en moyenne. En 1950, le système comptait 30000 étudiants; en 1960, 76000; en 1970, il dépassait les 271000 , en 1980, les 935000 , tandis qu'en 1990, ils étaient 1250000 et qu'ils dépassaient $1718000^{1}$ en 2000 . De nos jours ${ }^{2}$, le SESM accueille près de 2538000 étudiants répartis en 1892 institutions ${ }^{3}$.

Un regard sur l'ensemble des chiffres disponibles permet de noter que près de la moitié du SESM s'est développée ces quinze dernières années, ce qui conduit à affirmer qu'il s'agit d'un système d'enseignement extrêmement jeune, novateur et plein de vitalité. Dans ces conditions, pourquoi est-il souvent perçu différemment? De même, compte tenu de la continuité avérée des politiques publiques en matière d'enseignement supérieur et des diverses actions menées par l'ensemble des institutions ces dernières années, pourquoi imagine-t-on que la qualité de l'enseignement supérieur et l'intérêt porté par la société à la recherche universitaires ne répondent pas aux attentes réelles?

\footnotetext{
* Article traduit par Nuria Aligant.

1. OCDE (1997). Reviews of National Policies for Education: Mexico, Higher Education. OECD, Paris.

2. OCDE (2006). Thematic Review of Tertiary Education. Country Background Report for Mexico. OECD, Paris.

3. L'analyse de ces chiffres doit également tenir compte des dimensions géographiques du Mexique (une superficie de deux millions de $\mathrm{km}^{2}$ ) et de son poids démographique (une population de 105 millions d'habitants).
} 
Les réponses à ces questions sont multiples et obéissent à divers facteurs souvent corrélés. Cependant, et d'un point de vue de la société de la connaissance, il convient d'en retenir deux principaux :

- malgré son développement remarquable, le système d'enseignement supérieur mexicain donne les signes d'un épuisement et d'une obsolescence inhérents à ceux d'un vieux système ${ }^{4}$;

- la recherche universitaire ne dispose pas de l'ossature nécessaire qui lui permettrait d'avoir une réelle répercussion sur l'enseignement; d'autre part, elle n'est pas dotée des dispositifs et des réseaux nécessaires pour favoriser l'innovation et le développement national.

\section{L'ENSEIGNEMENT SUPÉRIEUR AU MeXiQue}

L'enseignement supérieur mexicain est le fruit du métissage entre l'héritage de l'université de Salamanque, qui remonte à l'époque coloniale, l'influence positiviste française de la fin du XIX ${ }^{\mathrm{e}}$ siècle, certains "airs latino-américains" du début du siècle dernier et la puissance anglo-saxonne (de l'Angleterre comme des États-Unis) dont l'influence est apparue vers la moitié du $\mathrm{XX}^{\mathrm{e}}$ siècle au niveau du troisième cycle et de la recherche.

La fonction de professionnalisation du SESM est définie en référence à un modèle prédominant d'enseignement, considéré depuis longtemps comme étant le meilleur pour former les futurs professionnels : un cursus en cinq ans, basé sur les disciplines ou les pratiques liées à la future activité professionnelle. Cette prééminence se manifeste de nombreuses manières : dans le terme générique de «licenciatura», qui désigne ce niveau d'études et signifie que les étudiants obtiennent une licence, au sens littéral du terme, qui leur permet d'exercer leur métier (pour faire commerce de leurs connaissances) à l'issue de leurs études; les arts, les humanités et les sciences n'échappent pas à cette pratique; les programmes d'enseignement laissent peu (ou pas) de place à l'étude des disciplines ou thématiques autres que celles initialement choisies par les étudiants.

La vision centralisée, nationaliste et napoléonienne de l'université est très marquée, notamment à travers le rôle central qu'elle a joué, au cours du siècle dernier, dans le développement du système d'enseignement supérieur dans les universités nationales et celles des États; dans le rôle prépondérant que ces universités ont eu (et qu'elles cherchent à perpétuer); dans la formation des cadres dont le pays a besoin et dans la construction de l'identité et la préservation de la culture nationales; enfin, dans l'idée récurrente que l'enseignement

4. Blanco José (2007). «La universidad mexicana », Punto de Inflexión, Instituto Mexicano para la Competitividad, 220-223, México. 
supérieur est un bien public et que, par voie de conséquence, l'enseignement supérieur public est moral ou idéologiquement supérieur à celui dispensé par les universités particulières ou privées.

L'administration collégiale des universités se manifeste à travers les divers conseils, commissions et autres structures, autorités et dispositifs de gouvernance universitaire existants; par les critères qui définissent l'appartenance et l'intégration à ces instances et qui déterminent l'accès aux fonctions de direction. La combinaison de cette vision de l'administration avec celle de la fonction de professionnalisation de l'enseignement supérieur se traduit également dans l'organisation de la plupart des universités en facultés et en écoles.

La tension université-gouvernement est explicite dans l'adjectif autónoma que maintiennent, dans leur dénomination, bon nombre d'universités tant publiques que privées. C'est là une source de conflits permanents avec l'appareil de l'État lorsqu'il s'agit de définir qui, du gouvernement ou de l'université, a le pouvoir de déterminer les besoins de la société.

À ces modèles dominants, il convient d'ajouter le modèle d'enseignement-apprentissage proposé par la plupart des établissements. Ce modèle est contemporain des processus suivis par les quatre modèles déjà cités, mais il s'avère d'une importance capitale pour l'avenir du système d'enseignement supérieur mexicain, tant en raison de sa nature propre que du point de vue de la société de la connaissance.

Le modèle pédagogique prédominant dans le système d'enseignement supérieur mexicain s'articule autour des connaissances et des contenus et se caractérise par un enseignement circonscrit à la salle de cours, magistral et basé sur des programmes encyclopédiques et rigides.

Le troisième cycle, en particulier le doctorat, résulte également de deux influences extérieures très marquées : d'une part, l'arrivée au Mexique des intellectuels espagnols après la guerre civile en Espagne, qui a fortement relancé la tradition humaniste mexicaine; d'autre part, l'obtention des premiers doctorats par des étudiants mexicains dans des universités européennes ou américaines. Ces deux facteurs conjugués ont été déterminants dans le développement des études doctorales, dans la recherche scientifique et dans l'émergence d'un corps d'enseignants universitaires au sens moderne du terme. Très vite, cet enseignement a adopté le modèle des États-Unis.

Jusqu'en 1960, le nombre de docteurs diplômés de l'enseignement supérieur mexicain se situait autour de $10 \%$, la majorité étant issue de l'Université nationale autonome de Mexico (UNAM). Les centres de recherche scientifique étaient peu nombreux et particulièrement orientés vers une recherche descriptive et peu vers une recherche expérimentale. Le corps enseignant universitaire était majoritairement composé de professionnels érudits qui, outre leur propre activité professionnelle, enseignaient dans les universités. À cette époque, l'enseignement à temps complet était davantage l'exception que la règle. 
Le développement des doctorats et de la recherche universitaire est devenu en peu de temps l'activité prédominante des principales institutions du SESM. L'impact des cycles de doctorat sur le développement de l'enseignement supérieur et de la science a été au Mexique très significatif et c'est devenu le mode de formation normal de l'enseignant universitaire mexicain.

\section{Croissance ET DIVERSIFICATION}

Actuellement, le système d'enseignement supérieur mexicain est constitué d'une communauté d'institutions très large et très diversifiée qui présente une hétérogénéité marquée en termes de nombre d'étudiants, d'objectifs, de modalités de fonctionnement et de financement. Des établissements d'enseignement supérieur de très grande taille, accueillant des milliers d'étudiants et proposant des programmes de premier et deuxième cycle (licence et professionnel associé) et de troisième cycle (spécialisation, maîtrise et doctorat) dans toutes les disciplines de la connaissance, coexistent avec d'autres établissements qui comptent moins de cinq cents étudiants et n'offrent des programmes que pour un seul niveau d'études. S'il est vrai que les premiers sont essentiellement composés d'une majorité d'universités publiques fédérales et des États ${ }^{5}$, les seconds se caractérisent par l'existence d'un grand nombre d'universités privées de création récente. Cependant, l'hétérogénéité qui règne dans les divers ensembles ou soussystèmes de ces institutions se reproduit à l'intérieur de plusieurs d'entre elles.

Par rapport au nombre total d'étudiants, les universités fédérales comptent $12 \%$ de licence, $25 \%$ de maîtrise et $40 \%$ de doctorat; les universités des États $33 \%, 25 \%$ et $20 \%$; les universités privées $33 \%, 45 \%$ et $18 \%$.

Ces institutions ont généralement pour mission le développement de la recherche, mais seule une centaine d'entre elles s'y emploie réellement. En revanche, les programmes de troisième cycle sont plus harmonieusement répartis et se multiplient, y compris ceux ayant trait aux études doctorales. En réalité, plusieurs institutions - dont certaines jouissant d'une excellente réputation ne proposent que des études de troisième cycle.

La dimension considérable atteinte actuellement par le système d'enseignement supérieur mexicain est étroitement liée au taux de développement démographique du pays dans la seconde moitié du $\mathrm{XX}^{\mathrm{e}}$ siècle ainsi qu'à la demande croissante d'enseignement supérieur : tandis qu'en 1950, $1 \%$ seulement des jeunes âgés de dix-neuf à vingt-trois ans accédait à l'université, $25 \%$ des jeunes actuels suivent des études supérieures. Pourtant, dans la même période, la population a été multipliée par quatre.

5. Politiquement, le Mexique est une République fédérale qui compte 31 États et un district fédéral, siège de la capitale du pays où résident les pouvoirs fédéraux. Certains États comptent plus d'une université d’État. 
Le système d'enseignement supérieur au Mexique

Nombre et pourcentage d'institutions et d'étudiants par sous-système

\begin{tabular}{|l|rr|rc|}
\hline Sous système & Institutions & \% & Étudiants & \% \\
\hline Universités fédérales & 4 & 0,2 & 307778 & 12,1 \\
\hline Universités d'État & 46 & 2,4 & 785917 & 31,0 \\
\hline Instituts technologiques & 211 & 11,2 & 325081 & 12,8 \\
\hline Universités technologiques & 60 & 3,2 & 62726 & 2,5 \\
\hline Universités polytechniques & 18 & 1,0 & 5190 & 0,2 \\
\hline Universités interculturelles & 4 & 0,2 & 1281 & 0,05 \\
\hline Écoles normales publiques & 249 & 13,2 & 92041 & 3,6 \\
\hline Institutions privées & & & & \\
de l'enseignement supérieur & 995 & 52,6 & 776555 & 30,6 \\
\hline Écoles normales privées & 184 & 9,7 & 54267 & 2,1 \\
\hline Centres publics de recherche & 27 & 1,4 & 2801 & 0,11 \\
\hline Autres institutions publiques & 94 & 5,0 & 124609 & 4,9 \\
\hline Total & $\mathbf{1 8 9 2}$ & $\mathbf{1 0 0 , 0}$ & $\mathbf{2 5 3 8 2 5 6}$ & $\mathbf{1 0 0 , 0}$ \\
\hline
\end{tabular}

Source : OCDE (2007). Thematic Review of Tertiary Education, Country Note Mexico, OCDE, Paris.

Pour répondre à cette demande galopante, beaucoup d'universités ont élargi leurs cursus de formation d'enseignement supérieur et pré-universitaire ${ }^{6}$. Cet élargissement a supposé, dans de nombreux cas, l'embauche d'enseignants peu préparés et peu motivés ainsi que la multiplication des instances universitaires de gestion et d'administration.

Les conséquences ont été lourdes : augmentation des coûts liés au financement du système d'enseignement, développement des syndicats universitaires et corporatisme institutionnel, mise en question de la qualité de l'université publique et accroissement des inscriptions dans des institutions privées pour les jeunes des classes sociales les plus aisées.

Face à cette situation, le gouvernement fédéral a intensifié la diversification des institutions et ses exigences en matière de financements des universités publiques. Il a élaboré et mis en œuvre des programmes pour augmenter les salaires et améliorer le profil académique des enseignants, proposé des initiatives en faveur de l'élaboration et du développement de projets institutionnels, encouragé la création de mécanismes d'évaluation (externe) de la qualité de l'enseignement supérieur et incité à la création d'universités privées.

6. De nos jours, plusieurs universités publiques et certaines universités privées proposent des études préuniversitaires, d'enseignement secondaire supérieur pour les jeunes âgés de quinze à dix-huit ans. 
Au cours du temps, la continuité des politiques publiques en matière d'enseignement supérieur ainsi que le respect des règles et des dispositifs mis en œuvre ont diminué la tension qui existait entre universités publiques et gouvernement fédéral; les progrès réalisés dans les domaines transférés par celui-ci aux universités sont devenus de plus en plus manifestes. Dans certains cas, les résultats obtenus ont amplement dépassé ceux affichés par des pays disposant de davantage de ressources et d'une tradition académique ${ }^{7}$.

Parmi les progrès les plus significatifs, il convient de citer : l'intégration des femmes, l'accroissement du nombre d'enseignants à plein temps avec un profil académique adapté, l'approche plus systémique du développement institutionnel et le rapport aux modes de financement, la multiplicité des dispositifs d'évaluation et de certification des programmes d'études et des institutions, ainsi que l'utilisation croissante de l'évaluation des apprentissages moyennant des tests standard mis en œuvre au début et à la fin des études universitaires, le développement du troisième cycle et de la recherche universitaire.

\section{L'ENJEU DE LA QUALIFICATION}

Le développement significatif du système d'enseignement supérieur mexicain a eu de nombreux avantages mais il a également eu un coût : la focalisation sur les éléments quantitatifs et la défense des modèles existants se sont faites au détriment de l'innovation pédagogique et des attentes de la société. Durant un demi-siècle, le système d'enseignement supérieur s'est focalisé sur son propre développement et a investi sur l'amélioration de ce qu'il mettait déjà en œuvre, sans prendre vraiment la mesure des bouleversements constants de son environnement. Ce repli sur lui-même a certainement entravé la réflexion et les initiatives nécessaires pour atteindre les objectifs premiers des missions qui lui étaient dévolues. Pendant plus d'un demi-siècle, le système a travaillé, souvent de façon intense, sur la base du même modèle éducatif, le reproduisant encore et encore et s'opposant - détruisant, mettant un terme ou minimisant à tout écart susceptible de l'en éloigner.

Mais le pays et le monde ont beaucoup changé ces dernières décennies. La dynamique de la connaissance au niveau mondial montre que les pays ont besoin de disposer d'un plus grand nombre de spécialistes et d'une population mieux préparée. Dans le cas du Mexique, cela se traduit par la nécessité impérieuse d'augmenter très rapidement le nombre de jeunes qui suivent des études doctorales ou de maîtrise (maestria) dans l'ensemble des domaines ainsi que le nombre des étudiants dans le premier cycle universitaire. Cela suppose que les étudiants soient mieux préparés et capables d'une plus grande autonomie dans

7. ANUIES (2005). Acciones de transformación de las universidades públicas mexicanas 1994-2003. Asociación Nacional de Universidades e Instituciones de Educación Superior, México. 
leurs apprentissages afin de pouvoir s’informer sur les évolutions constantes dans d'autres domaines que le leur, ce qui leur permettrait d'établir les passerelles nécessaires entre plusieurs domaines de connaissances.

Parvenir rapidement à cet objectif constitue un défi extraordinaire pour un pays de la taille du Mexique qui compte moins de 2000 docteurs par an issus de ses établissements supérieurs. Les étudiants mettent en moyenne plusieurs années de plus qu'il ne faudrait à terminer leurs études universitaires et une majorité d'entre eux n'a pas l'opportunité, au cours de son cursus universitaire, d'approfondir d'autres champs disciplinaires que ceux qui lui sont propres, sans compter que les passerelles entre filières ne sont pas aisées.

Les bouleversements intervenus sur le marché du travail ainsi que dans les profils professionnels requis pour accéder à des postes d'encadrement montrent que les compétences d'antan ne sont pas nécessairement celles qui seront exigées dans le futur et que le secteur productif de la société de la connaissance requiert et récompense de nouvelles compétences et savoir-faire. Au Mexique, pays dans lequel seuls $16 \%$ des professionnels actuels ont suivi un enseignement universitaire, $85 \%$ des étudiants se regroupent autour de 25 filières d'études et le nombre de personnes qui arrivent sur le marché du travail sans diplôme supérieur reste toujours supérieur au nombre de titulaires. Former davantage de jeunes dans de nouveaux domaines de la connaissance, tout en leur transmettant de nouveaux savoir-faire, est un défi considérable.

Le développement soutenu des technologies de l'information et de la communication modifie l'activité humaine et rend «l'alphabétisation informatique» aussi importante, de nos jours, que la lecture ou l'écriture. Par conséquent, il paraît essentiel, si l'on veut que la société soit davantage informatisée, d'introduire les nouvelles technologies et l'utilisation des moyens de communication dans les cursus universitaires. Par ailleurs, il est tout aussi important que les contenus des enseignements soient modifiés. Le modèle pédagogique proposé par la plupart des établissements mexicains est dépassé. L'information y est réduite et se trouve concentrée dans un espace et un temps définis, tandis que dans le monde actuel et futur, elle est abondante et s'obtient en tout temps et en tout lieu (ou presque). Souvent, les enseignants et les programmes d'étude actuels attendent des étudiants qu'ils mobilisent leur potentiel mental pour "prendre des notes» et mémoriser, au lieu de leur apprendre à se servir de leur intelligence, de leur imagination et de leur sensibilité pour résoudre des problèmes, trouver des alternatives et explorer d'autres chemins, tout en s'appuyant sur les nouveaux équipements et les bases de données à leur disposition. Ceci leur permettrait de développer des connaissances et de se les approprier au lieu de répéter inlassablement celles de leurs maîtres. Dans ces conditions, il n'est pas étonnant que peu d'étudiants du supérieur deviennent entrepreneurs, promoteurs, concepteurs, scientifiques ou artistes, et que beaucoup d'entre eux ne soient que des employés. 
La nécessité d'avoir des populations de mieux en mieux préparées, afin de préserver ou d'accroître les atouts concurrentiels qu'elles possèdent, ont conduit bon nombre de pays à réfléchir à de nouvelles méthodes plus efficaces qui facilitent et attirent le plus grand nombre d'étudiants vers des niveaux plus élevés d'études et ce, dans un large éventail de domaines de spécialité. Pour y parvenir, ils ont accepté de modifier la structure, le sens et l'organisation de leurs vieux systèmes d'enseignement supérieur et de recherche. Le cas le plus patent, connu sous le nom de Processus de Bologne, est celui des pays européens. Le chemin entrepris, et qui a pour objectif de revigorer l'enseignement supérieur et de développer l'innovation et la recherche scientifique dans la région, devra rapprocher les différents systèmes d'enseignement supérieur en Europe du modèle anglo-saxon.

L'exemple le plus proche du Mexique, qui est celui du modèle des États-Unis, montre que seuls $2 \%$ d'inscrits dans l'enseignement supérieur suivent un cycle d'études tendant à l'obtention d'un diplôme dans une profession réglementée. En revanche, $98 \%$ suivent des d'études de premier cycle (deux ans, généralement dans des filières professionnelles), de second cycle (pour ceux qui suivent des enseignements généraux, non professionnels) ou bien de niveau III (maîtrises et doctorats).

Le fait que le système d'enseignement supérieur mexicain soit confronté aux difficultés caractéristiques d'un pays en développement - inégalité des chances éducatives selon les régions géographiques ou l'appartenance sociale, retard scolaire dans les niveaux précédant l'enseignement supérieur, faibles systèmes d'innovation, ressources financières limitées, activité scientifique et technologique réduite, normes et standards défaillants - renforce l'idée qu'il est urgent que le Mexique se dote d'un système d'enseignement supérieur efficace et pertinent adapté à son présent et surtout à son avenir.

Actuellement, il existe au Mexique des centaines d'instituts et d'universités technologiques et généralistes tout comme des universités et des institutions privées en mesure d'offrir des cursus universitaires autres que ceux qui sont traditionnellement proposés, tant en ce qui concerne la durée des études que les critères ou les méthodes d'enseignement. Cependant, l'offre d'études ainsi que la population étudiante ont tendance à rester concentrées au niveau de la licence. Par ailleurs, le nom ou le type d'établissements d'enseignement supérieur ne reflète pas toujours leurs objectifs, leurs missions, leur pédagogie, leur organisation et leur réalité; certaines universités agissent comme de vrais établissements de second degré et certains établissements de second degré sont de vraies universités. De même, il n'est pas toujours aisé de bien percevoir la distinction entre université polytechnique et université technologique, par exemple, ou bien entre un diplôme de "professionnel associé » et celui de «technicien supérieur universitaire».

En somme, la puissance et l'inertie des principales universités mexicaines et le prestige que la société accorde au diplôme de licence agissent comme autant 
d'entraves pour qu'une réelle diversification institutionnelle puisse avoir lieu; la plupart des institutions, en particulier les universités publiques, perpétuent un modèle obsolète, un discours public similaire à celui d'il y a trente ans et un modèle pédagogique traditionnel.

Cependant, l'organisation et les conditions actuelles rendent le système d'enseignement supérieur mexicain plus ouvert au changement qu'auparavant. En outre, les réponses du système aux attentes de la société montrent des défaillances et des contradictions aiguës, alors que les défis internationaux deviennent de plus en plus singuliers et déterminants pour le pays et pour son système d'enseignement supérieur.

\section{L'AVENIR}

Le Mexique connaît une transformation démographique majeure ${ }^{8}$ qui entraîne un développement de la population active et une diminution du nombre de personnes économiquement dépendantes de cette dernière. Profiter de ce contexte requiert la mise en place de mesures appropriées. En effet, sans un développement de la productivité individuelle, l'arrivée sur le marché d'un plus grand nombre de personnes n'aura pas nécessairement les retombées escomptées sur le développement de l'épargne ou l'investissement afin d'accroître la richesse individuelle et collective. Le système d'enseignement supérieur mexicain est appelé à jouer un rôle de premier ordre dans l'information et la communication, dans les domaines de la formation, l'évaluation et la certification des travailleurs concernant les connaissances, les savoir-faire et savoir-être que le nouveau contexte exige.

Certes, l'intégration du Mexique dans la société de la connaissance passe par l'adoption de nouveaux objectifs mais exige également que l'ensemble des institutions du supérieur s'engage dans un processus de réflexion sur la nature, l'organisation, l'attrait et la pertinence de l'offre éducative ainsi que sur la capacité, l'orientation, l'impact et la place qu'elles souhaitent accorder à la recherche. Par conséquent, elles doivent questionner la manière dont elles remplissent les missions qui leur sont traditionnellement dévolues.

Améliorer le niveau général de formation de la jeunesse, faciliter l'accès aux études supérieures des populations en développement, accroître l'efficacité, l'indépendance et l'initiative personnelles de tous les étudiants et développer une plus grande spécialisation et diversification des diplômés exige des changements profonds des méthodes et des modes d'organisation, d'orientation et d'enseignement. Certains de ces changements ont été conduits dans les universités publiques technologiques et polytechniques et dans des établissements privés

8. En 1965 , le taux de croissance démographique était de 3,5\%; actuellement, il est de $1 \%$. 
comme l'Université Tec Milenio. Par ailleurs, d'autres universités publiques et privées traditionnelles réfléchissent également à l'opportunité d'engager des bouleversements considérables dans leurs pratiques d'enseignement et dans leur organisation.

Beaucoup d'universités s'intéressent aux phénomènes d'internationalisation de l'enseignement supérieur, établissant des accords de partenariat avec des universités d'autres pays et régions du monde, ce qui favorise la mobilité de leurs enseignants et étudiants, la réalisation conjointe de troisièmes cycles et de projets de recherche ou la collaboration à des programmes d'enseignement à distance. Certaines suivent le Processus européen de Bologne avec attention et réfléchissent en privé sur l'opportunité d'abandonner les programmes d'études longs, rigides, encyclopédiques et spécialisés qui caractérisent l'enseignement supérieur mexicain et qui s'appliquent sans distinction aux arts, aux humanités et aux sciences, au profit de programmes d'enseignement plus courts, généraux et flexibles. Le Mexique est confronté aux mêmes doutes que ceux qui se sont manifestés en Europe : quels devront être les principaux contenus et objectifs du premier cycle d'études? Et si les filières professionnelles se concentrent uniquement sur une ou deux années après ce premier cycle, comment garantir que l'étudiant diplômé possède les connaissances, compétences et savoir-faire attendus de lui à l'issue de ses études?

D'autres universités vont au-delà de ces réflexions et parlent de modifier le modèle pédagogique centré sur le cours magistral, la salle de cours et les contenus disciplinaires uniques par un autre tourné davantage vers l'abondance d'information caractéristique de notre époque, les technologies et les compétences. Elles s'interrogent également sur les dispositifs à mettre en place pour que les enseignants deviennent des médiateurs capables de transmettre à leurs étudiants les connaissances, les concepts, les méthodes et les techniques les plus récentes dans les diverses disciplines enseignées; d'encourager leur créativité, leur esprit critique et l'auto-apprentissage; ainsi que leur intérêt pour la recherche scientifique et pour l'innovation ${ }^{9}$.

Dans ce domaine, les actions sont encore peu développées et, souvent, ne font pas l'objet de publicité afin de ne pas susciter l'inquiétude (et l'opposition) des étudiants et des enseignants. Cependant, de nombreuses universités ont déjà participé à des projets internationaux qui avaient pour objectif de mener une réflexion sur l'avenir de l'université au Mexique et en Amérique latine, à l'image de ce qui est mis en ouvre en Europe. L'une de ces actions a eu pour objectif d'élaborer une proposition visant le transfert de crédits et de compléments de diplômes universitaires, en harmonie avec des correspondants

9. Le meilleur exemple est celui de l'Université Veracruzana, une université publique d'État, composée de plusieurs sites, qui s'est engagée dans une approche différente de ses programmes d'études et qui a décidé d'adhérer au Processus de Bologne. 
européens ${ }^{10}$. Si cette proposition était généralisée, elle faciliterait la mobilité internationale des étudiants mexicains et permettrait le transfert et/ou l'accumulation de crédits pour les universitaires mexicains.

Les inquiétudes concernant le troisième cycle et la recherche scientifique portent essentiellement sur la question du développement du nombre d'étudiants inscrits et de l'atténuation de sa forte orientation académique actuelle, sans que cela pénalise sa qualité. Le troisième cycle devrait être plus attractif et permettre à plus d'étudiants d'obtenir un diplôme; il devrait les préparer solidement à être en mesure d'apporter des solutions et de développer des alternatives aux multiples problèmes auxquels la société mexicaine est confrontée, sans alourdir des cursus et des apprentissages dont la durée est sans doute excessive.

Par ailleurs, il convient également de réfléchir à la façon de rendre plus attractives, pour les jeunes doctorants, les recherches sur des problématiques liées au monde de la production et de l'industrie. Il y a quelques années, les exemples du Japon et de la Corée ont permis de comprendre qu'il était envisageable de s'approprier rapidement la connaissance; c'est actuellement le cas de l'Inde et de la Chine. Le Mexique aspire également à trouver le moyen de dynamiser sa société et de construire des systèmes d'enseignement supérieur et d'innovation solides.

10. En février 2006, le groupe de pays signataires de la Convention pour la reconnaissance des titres et diplômes de l'Institut international de l'Unesco pour l'enseignement supérieur en Amérique latine et dans les Caraïbes (IESALC) a reconnu et approuvé la proposition visant la mise en œuvre d'un "système de crédits académiques (SICA) " et d'un «complément au titre (CAT)» élaborée par les universitaires qui participent au projet Six métiers autour de quatre axes: un dialogue universitaire $(6 \times 4$ UEALC). Voir http://www.6x4uealc.org/. 